\title{
Research of Absolute Sensorless Cutting Force Induced Error Compensation System
}

\author{
Xiaolong Shen ${ }^{1, a}$, Meilin $\mathrm{Li}^{2, b}$ and Nanlin $\mathrm{Yu}^{1}$ \\ ${ }^{1}$ Hunan Industry Polytechnic, Changsha, 410208, China \\ ${ }^{2}$ Hunan Urban Construction College, Xiangtan, 411101, China \\ ashenXL64@163.com, 'limeilin0731@yeah.net
}

Keywords: Absolute sensorless, CFIE, Artificial neural network, Compensation system.

\begin{abstract}
Based on the on the already established compensations systems, mechanistic modeling, a sensorless systems is attained. In this Artificial Neural Networks are used to learn through mimicking of the machining parameters with reference to the existing Compensations systems, Finally the performance of the Network is tested and deductions are made for future sensorless systems. After the equilibrium between the output and desired values, then the ANN is fully trained and ready for deployment, Designed net works can be conveniently implemented on general computers as well as independent processors.
\end{abstract}

\section{Introduction}

Mechanistic modeling has been used to predict the tool thermal analysis, tool life estimations, chatter prediction and tool integrity. Also significant efforts have been devoted to understanding of the forces in metal cutting[1-2] and many experimental approaches have been proposed and implemented. Nevertheless, they have not been exploited the fore-achieved experiences in the fields of developing CFIE (Cutting Force Induced Errors) compensation systems.

Clearly analytical models are favored because they are easy to implement and convey more insight of their metal cutting characteristics. The Milling process in focus for procedure is not single point cutting but rather multi-point cutting process, Though it is multi-point cutting process still is based on single point cutting principles. Briefly, milling is like multitude of single point cutting processes taking place at the same time and same stations.

\section{Mechanistic Modeling of Metal Cutting process}

Since the generation of cutting force sorely occurs at the touching point of the workpiece and cutting tool, Therefore mechanistic modeling can be applied to determine the components of cutting force based on the physical cutting conditions and machine tool parameters. This includes machining parameters, cutting tool geometry, undeformed chip thickness and workpiece information.

In order to model the milling process, the power expression[3] was utilized to determine the cutting force. Whereby, tangential cutting force component $\left(F_{c}\right)$ in face milling is expressed as follow:

$$
F_{C}=C_{F_{C}} \cdot a_{e}{ }^{\left(C_{F_{e}}\right)} \cdot f^{\left(C_{F_{f}}\right)} \cdot a_{p}{ }^{\left(C_{F_{p}}\right)} \cdot d^{\left(C_{F_{d}}\right)} \cdot z_{e}
$$

Although the $\mathrm{x}, \mathrm{y}$ and $\mathrm{z}$ components are affected by the resolving angle factor and geometries of the cutting tool, all ale still proportional to the radial component of cutting force[4-5]. This proportionality is represented by constants as illustrated in equation (2).

$$
\left.\begin{array}{l}
F_{C x}=C_{F_{C}} \cdot a_{e}{ }^{\left(C_{F_{e}}\right)} \cdot a^{\left(C_{F_{c}}\right)} \cdot a_{p}{ }^{\left(C_{F_{p}}\right)} \cdot d^{\left(C_{F_{d}}\right)} \cdot z_{e} \cdot K_{F_{x}} \\
F_{C y}=C_{F_{C}} \cdot a_{e}{ }^{\left(C_{F_{e}}\right)} \cdot a^{\left(C_{F_{c}}\right)} \cdot a_{p}{ }^{\left(C_{F_{p}}\right)} \cdot d^{\left(C_{F_{d}}\right)} \cdot z_{e} \cdot K_{F_{y}} \\
F_{C z}=C_{F_{C}} \cdot a_{e}{ }^{\left(C_{F_{e}}\right)} \cdot a^{\left(C_{F_{C}}\right)} \cdot a_{p}^{\left({ }^{\left(C_{F_{p}}\right)} \cdot d^{\left(C_{F_{d}}\right)} \cdot z_{e} \cdot K_{F_{z}}\right.}
\end{array}\right\}
$$




\section{Experimental setup and data acquisition}

The current based sensory system is already available as per the previous procedure. Based on this already exiting model, the cutting forces predicting model are established. This model just is used for determining of the Machine machining variables (Speed, feed) and cutting parameters. Finally the modeling process is accomplished by supervised Artificial Neural Network. Whereby the ANN is training by information attained from experimental proceedings as shown in fig.1.

The establishment of sensorless system is based on the already exiting indirect monitoring CFIE compensation systems[6]. And the relationship is achieved by engaging the current sensor while machine trial specimen and the corresponding values of currents at different depth of cut are recorded.

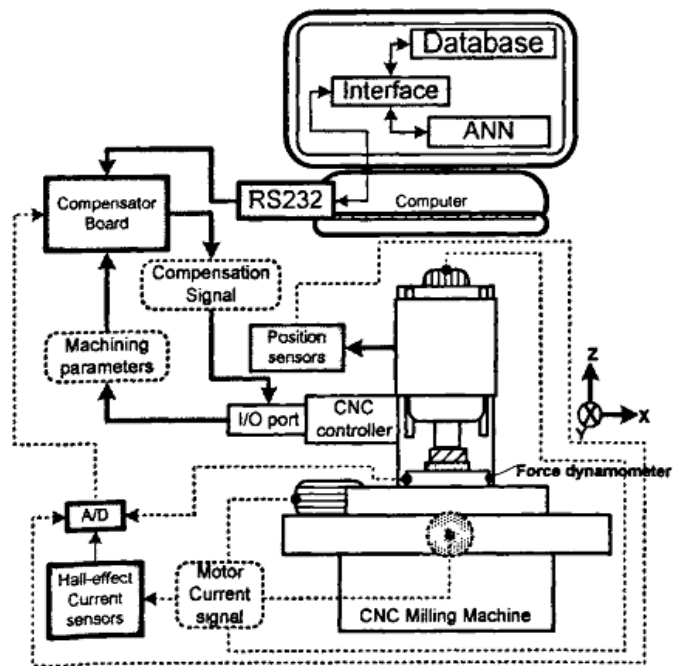

Fig.1 Setup for the Sensorless system

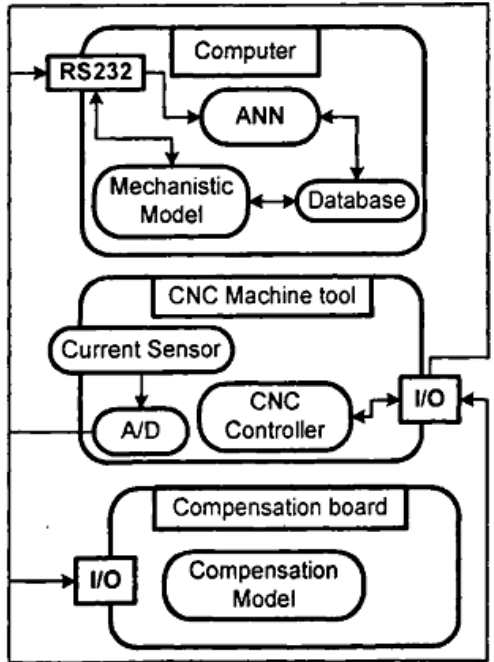

Fig.2 Experiment Procedural interpretation

As a result, this experimental setup does not basically vary from the previous one apart from additions of Neural Network Programming environment. The Neural Network Programming environment is based on the Simulink ToolBox in MatLab environment. As demonstrated in fig.2, the composition of the system includes: Compensation board; CNC controller; Computer. The Compensation board has a compensation model embedded in it, while the CNC controller is part of the CNC machine tool. The Personal Computer runs the Artificial Neural Network (ANN), Database, and mechanistic model.

Based on the values of the current sensors, the corresponding cutting force is predicted by the compensation model, and then these values are dispatched to computer through the RS232 interface. As well, the computer acquires modeling necessary machining parameters from the CNC controller via I/O port of the CNC machine tool. By considering the Cutting tools specifications, cutting parameters and material information sufficiently provide conditions for supervising the learning of the ANN.

Finally the mechanistic model is achieved and stored which can be fetched by the updated Mechanistic model. After sufficient experiments are done, then the model for the sensorless model is accomplished and ready for deployment.

\section{Neural Network Programming and training}

The basic Steps of ANN programming are namely: Obtaining Nonstandard Network topology; Conversion to Standard Neural Network; Network Training and Architecture Simplification; and Testing of Network Performance. The basic idea behind the concept is to develop neural networks with guidance of the available knowledge on the investigated process[7-8]. 
In order to obtain a standard network topology ANN architecture for power expression, the inputs are first processed through natural log function. Followed by weights multiplication to represent the power expression in equation (3) to a form as follows:

$$
\left.\begin{array}{l}
\operatorname{In}\left(F_{c x}\right)=\left[\operatorname{In}\left(C_{F_{C}}\right)+\operatorname{In}\left(K_{F_{x}}\right)+\operatorname{In}\left(z_{e}\right)\right]+C_{F_{e}} \operatorname{In}\left(a_{e}\right)+C_{F_{f}} \operatorname{In}(f)+C_{F_{p}} \operatorname{In}\left(a_{p}\right)+C_{F_{d}} \operatorname{In}(d) \\
\operatorname{In}\left(F_{c y}\right)=\left[\operatorname{In}\left(C_{F_{C}}\right)+\operatorname{In}\left(K_{F_{y}}\right)+\operatorname{In}\left(z_{e}\right)\right]+C_{F_{e}} \operatorname{In}\left(a_{e}\right)+C_{F_{f}} \operatorname{In}(f)+C_{F_{p}} \operatorname{In}\left(a_{p}\right)+C_{F_{d}} \operatorname{In}(d) \\
\operatorname{In}\left(F_{c z}\right)=\left[\operatorname{In}\left(C_{F_{C}}\right)+\operatorname{In}\left(K_{F_{z}}\right)+\operatorname{In}\left(z_{e}\right)\right]+C_{F_{e}} \operatorname{In}\left(a_{e}\right)+C_{F_{f}} \operatorname{In}(f)+C_{F_{p}} \operatorname{In}\left(a_{p}\right)+C_{F_{d}} \operatorname{In}(d)
\end{array}\right\}
$$

Through inputting parameters of cutting conditions into neutrons of supervised Artificial Neural Network (ANN), the model is attained. The learning process is achieved through backward propagation algorithms by mimicking the examples and hence optimally adjusting the weights accordingly.

$$
\begin{aligned}
& \text { Where: } \quad p=\left\{a_{e}, f, a_{p}, d, z_{e}\right\}, \quad R=5, \quad W=\left[\begin{array}{lllll}
W_{x, a_{e}} & W_{x, f} & W_{x, a_{p}} & W_{x, d} & W_{x, z_{e}} \\
W_{y, a_{e}} & W_{y, f} & W_{y, a_{p}} & W_{y, d} & W_{y, z_{e}} \\
W_{z, f} & W_{z, f} & W_{z, a_{p}} & W_{z, d} & W_{z, z_{e}}
\end{array}\right], \quad S=3, \\
& b=\left\{K_{C_{x}}, K_{C_{y}}, K_{C_{z}}\right\}, \quad F_{x y z}=\left\{F_{C x}, F_{C y}, F_{C z}\right\}
\end{aligned}
$$

The vector $\mathrm{P}$ represents the inputs to the neutrons, while $\mathrm{W}$ is the matrix of weights representing a set of powers in equation (3). Furthermore, b stands for the bias vector that represents the combination effect of workspace material properties, cutting geometry and cutting conditions. As shown in fig.3, inputs are converted to natural logs and multiplied by weights and then summed up. After that, in accordance with sigmoid the activation function, the sums are processed and reverted to original power form through exponential function. Finally the magnitude of the respective $\mathrm{x}, \mathrm{y}$, and $\mathrm{z}$ components are achieved.

To train the standard ANN representing ideal machining process, a training set is first formed by recording the fundamental motor currents induced by cutting force variations. The same face milling tool of $20 \mathrm{~mm}$ of diameter fitted with inserts of tungsten Carbide was used on both carbon steel and cast steel specimen at different depth of cuts and width of cut. The shape of the specimens used in the data acquisition experiments are shown in fig.4. The specimens where prepped in with geometry that implicated varying depth of cut and had different width. Implying that in one fixture of the worktable for one specimen will be able represent a variation of depth of cut in without the actual position of the tool in the $\mathrm{z}$ direction hence avoiding location errors from mounting of the workpiece.

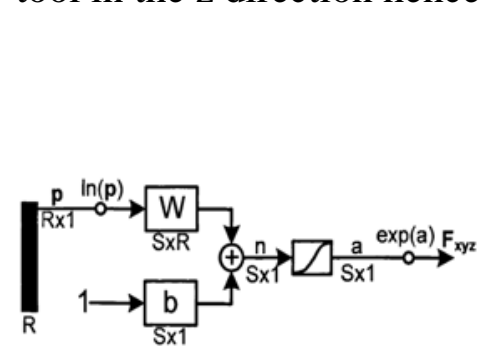

Fig.3 Artificial Neural Architecture

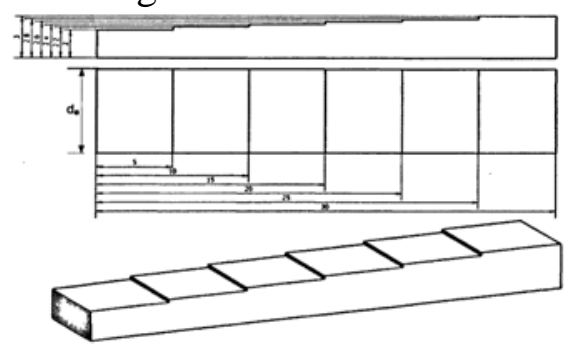

Fig.4 Test block for ANN training experiments

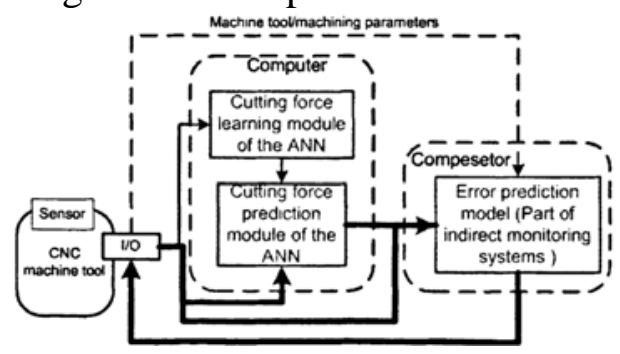

Fig.5 Realization of Sensorless compensation systems

The data acquired is used to train the ANN system for the following situations:

Dept of cut $\left(\mathrm{a}_{\mathrm{p}} / \mathrm{mm}\right)=\{0.8,1.0,1.2,1.4,1.6,1.8\}$

Feed per tooth $(\mathrm{f} / \mathrm{mm} / \mathrm{rev})=\{0.066,0.072,0.087,0.1\}$

Number of teeth $(\mathrm{z})=\{4\}$

Width of cut $\left(\mathrm{a}_{\mathrm{e}} / \mathrm{mm}\right)=\{10,15,20\}$

Cutting force ranges for carbon steel are $\left(\mathrm{F}_{\mathrm{x}}, \mathrm{F}_{\mathrm{y}}, \mathrm{F}_{\mathrm{z}}\right)=\{(258.08 \sim 1882.78),(91.47 \sim 794.58)$,

(150.57 1091.76)\}

Cutting force ranges for cast iron are $\left(\mathrm{F}_{\mathrm{x}}, \mathrm{F}_{\mathrm{y}}, \mathrm{F}_{\mathrm{z}}\right)=\{(166.24 \sim 1036.48)$, (55.59 435.05),

(99.02 601.35)\} 
Through a series of training session based on the back-propagation supervised method, finally matrices and vectors for weights and biases respectively were attained.The matrix of the weights and vector of biases for the case of carbon steel are respectively as follows:

$$
\begin{aligned}
W_{\text {cns }} & =\left[\begin{array}{lllll}
1.103 & 0.747 & 1.002 & 0.997 & -1.299 \\
1.098 & 0.748 & 0.997 & 1.006 & -1.299 \\
1.100 & 0.751 & 0.995 & 1.004 & -1.301
\end{array}\right] \\
b_{\text {cns }} & =\{316.91,814.92,452.73\}
\end{aligned}
$$

Similarly the matrix of the weights and vector of biases for the case of cast iron are respectively as follows:

$$
\begin{aligned}
W_{\text {cns }} & =\left[\begin{array}{lllll}
1.003 & 0.737 & 0.902 & 0.997 & -0.999 \\
0.998 & 0.738 & 0.897 & 1.006 & -0.999 \\
1.000 & 0.741 & 0.895 & 1.004 & -1.001
\end{array}\right] \\
b_{\text {cns }} & =\{185.41,476.77,264.87\}
\end{aligned}
$$

Finally the established values of cutting forces are used to calculate the cutting force induced error based on a predetermined model basing on the existing model of indirect monitoring system. The ANN prediction model is expressed in terms of $1^{\text {st }}$ order polynomial and successfully embedded into the compensator. As illustrated in fig.5, after the learning of the ANN is finished, then the achieved data is transferred to the prediction module. Then the error predictions model of the already existing indirect monitoring systems uses the predicted cutting force components to predict the induced error in real time. On the other hand the sensorless system is exploited in predicting failure occurrence of online sensors and improvement system reliability as well.

\section{Summary}

1) Generations of cutting forces occur solely at the touching point of the cutting tool and workpiece, Therefore mechanistic modeling is the most direct means of monitoring cutting force. This shortens the chain of monitoring hence fewer variables involved for easy modeling, accuracy and reliability.

2) Through experiments, data of the motor current and other machining information are used to determine the cutting forces. The machining, cutting tool and workpiece information are used to generate forward propagation in the network.

\section{Acknowledgements}

This project is supported by Science and Technology Fund of Hunan Provincial Science and Technology Department (No.2011GK3090), and 2012 College Research Project of Hunan Industry Polytechnic Grant (No. GYKYZ2012010).

\section{References}

[1] Min Xu, Robert B. Jerard, Barry K. Fussell, Energy Based Cutting Force Model Calibration for Milling, Computer-Aided Design\&Applications, Vol. 4 (2007).

[2] X.L. Shen, Y.X. Luo, L.X. Zhang and H. Long: Adv. Mater. Res. Vol. 156-157(2011), p. 1582-1585.

[3] Jeffrey L. Stein, Kunsoo Huh, Monitoring Cutting Forces In Turning -A Model Based Approach, Transactions of the ASME, Vol. 124 (2002).

[4] J.F.G. Oliveira, T.V. Franca and J.P. Wang: CIRP Ann. Vol. 57 (2008), p. 329-332.

[5] X.L. Shen, L.X. Zhang, H.Long and Z.X. Zhou: Appl. Mech. Mater. Vol. 34-35 (2010), p. 1936-1940.

[6] H.L. Liu, B. Li, H.M. Shi et al., Embeded-system of position accuracy evaluation and error compensation of CNC machine, J. Huazhong Uni. Sci. Technol. 32 (2004), p.31-33. 
[7] X.L. Shen, L.X. Zhang, C.G. Ren and Z.X. Zhou: Adv. Mat. Res. Vols. 97-101 (2010), p. 2053-2057.

[8] G.L. Samuel and M.S. Shunmugam: Precision Eng. Vol. 24 (2000), p. 251-263. 